\title{
Self-similar lifting and persistent touch-down points in the thin-film equation
}

\author{
Carlota M. Cuesta ${ }^{1}$, Hans Knüpfer ${ }^{2}$, and Juan J. L. Velázquez ${ }^{3}$ \\ ${ }^{1}$ Departamento de Matemáticas, Faculty of Science and \\ Technology, University of the Basque Country (UPV/EHU), \\ e-mail: carlotamaria.cuesta@ehu.eus \\ ${ }^{2}$ Institut für Angewandte Mathematik, \\ IWR, Universität Heidelberg, \\ e-mail: hans.knuepfer@math.uni-heidelberg.de \\ ${ }^{3}$ Institut für Angewandte Mathematik, Universität Bonn, \\ e-mail: velazquez@iam.uni-bonn.de
}

November 7, 2018

\begin{abstract}
We study self-similar solutions of the thin-film equation

$$
h_{t}+\left(h^{m} h_{x x x}\right)_{x}=0 \quad \text { in }\{(x, t): \quad h(x, t)>0\}
$$

with $m \in(0,4]$, that describe the lifting of an isolated touch-down point given by an initial profile of the form $h_{\text {in }}(x)=|x|$. This provides a mechanism for non-uniqueness of the thin-film equation with $m \in(2,4)$, since solutions with a persistent touch-down point also exist in this case. In order to prove existence of the self-similar solutions, we need to study a four-dimensional continuous dynamical system. The proof consists of a shooting argument based on the identification of invariant regions and on suitable energy formulas.
\end{abstract}

Keywords: self-similar solutions, thin-film equation, non-uniqueness AMS subject classification: 35K65, 34A34, 76D27

Short title: Self-similar lifting in the thin-film equation.

\section{Introduction}

We consider the family of thin-film equations

$$
h_{t}+\left(h^{m} h_{x x x}\right)_{x}=0 \quad \text { in }\{(x, t): \quad h(x, t)>0\}
$$


for $m \in(0,4]$. In particular, we study solutions that exhibit self-similar lifting of an initial profile with a touch-down point of the form $h_{\text {in }}(x)=a|x|(a>0)$ for $m \in(0,4]$. This situation is relevant for the merging process of two adjacent droplets. Our result also gives an explicit example of the known non-uniqueness of weak solutions exhibited by (11) as we explain below. Before we state our results and put them into context, let us recall some properties of (10).

The model (10) describes the spreading of a liquid film on a substrate with height profile $h(x, t)$ whose evolution is driven by capillary effects. It can be derived formally from the Navier-Stokes equation in the so-called regime of lubrication approximation. The exponent $m>0$ is determined by the precise boundary conditions imposed at the liquid-solid interface. In particular, the case $m=3$ is related to imposing a no-slip condition at this interface, while the case $m=2$ corresponds to a Navier-slip condition [22, 31. The case of more general mobility $m>0$ can be derived using certain generalized Navier-slip type conditions, see e.g. [1, 8, 20, 29. Furthermore, for $m=1$, (1) arises as the lubrication approximation of the Hele-Shaw flow [11].

Equation (11) is a degenerate fourth order parabolic equation, formulated on a domain with a free boundary. Note that, while the equation is parabolic on the positivity set of $h$, the parabolicity degenerates at the points where $h$ vanishes. Solutions of (1) formally satisfy a dissipation relation, namely, for positive smooth solutions $h>0$, it is given by (see e.g. 4)

$$
\frac{1}{2} \frac{d}{d t}\left(\int_{\mathbb{R}} h_{x}^{2} d x\right)+\int_{\mathbb{R}} h^{m} h_{x x x}^{2} d x=0 .
$$

In this paper we use the following notion of weak solution of (1), see also [3, 4, 7]:

Definition 1.1 (Weak Solution). Let $t_{0} \geq-\infty$ and let $I=\left[t_{0}, \infty\right)$. Then $h \in C^{0}(I \times \mathbb{R}) \cap L^{\infty}\left(I, H_{\mathrm{loc}}^{1}(\mathbb{R})\right)$ such that $h^{m / 2} h_{x x x}$ exists in the distributional sense and

$$
\int_{\mathbb{R}}\left|h^{m / 2} h_{x x x}\right|^{2} d x<\infty \quad \text { for a.e. } t>t_{0} .
$$

is called a weak solution of (11) if $h \geq 0$ and for all $\psi \in C_{c}^{\infty}(I \times \mathbb{R})$, we have

$$
\int_{I} \int_{\mathbb{R}} h \psi_{t} d x d s+\int_{I} \int_{\{h>0\}} h^{m} h_{x x x} \psi_{x} d x d s=0 .
$$

The function $h_{\mathrm{in}} \in C^{0}(\mathbb{R})$ is called initial data if $h(x, t) \rightarrow h_{\mathrm{in}}(x)$ as $t \rightarrow t_{0}$.

Remark 1.2 (Dissipation). The expression $h^{m / 2} h_{x x x}$ in (3) is understood in the distributional sense. We notice that this definition differs from the a.e. pointwise definition for $m \in(0,2)$. For example, for the cone $h(x)=|x|$ we have $h^{m} h_{x x x}=0$ a.e. $x \in \mathbb{R}$. However, understood in the distributional sense, we have $h_{x x x}=\delta_{0, x}$ (the derivative of a Dirac mass at zero). By an argument based on integration by parts, it follows that $h^{\frac{m}{2}} h_{x x x}=|x|^{m / 2} \delta_{0, x}$ is not welldefined if $m \leq 2$ and vanishes if $m>2$ (see e.g. [13]). This shows that 
$h(x)=a|x|$ with $a>0$ are stationary weak solutions of (1) in the sense of Definition 1.1 if and only if $m>2$.

Remark 1.3 (Contact angle). The Definition 1.1 of weak solutions does not include a contact angle condition. Including such a condition for weak solutions is not straightforward since their regularity is not sufficient to control the first derivative point-wise. We note that there are other definitions of weak solutions. In particular, there is one that requires additional regularity (which leads to the so-called entropy solutions) that implies zero contact angle (see, for instance, [3, 4, 17] -one space dimension- and [9, 12] -several space dimensions). There are also formulations of weak solutions for a prescribed non-zero contact angle. This is a more delicate issue, since the corresponding solutions are less regular. We refer the reader to [30] for $m=1$, and to [10] for a different notion of weak solution that modifies the energy (2) and is valid for $m \in(0,3)$.

In the context of (11), the rupture of a droplet into two droplets and, reversely, the merging of two droplets into a single droplet, corresponds to a topological change of the positivity set $\{h>0\}$. In this work, we consider the situation when the positivity set initially consists of two connected components which are separated at a single touch-down point in the specific case $h_{\text {in }}(x)=a|x|$. For this initial data and for $m \in(0,4)$, we show that there exist self-similar solutions which display lifting of the touch-down point. These solutions are weak solutions in the sense of Definition 1.1. For completeness, we also consider the case $m=4$ which corresponds to lifting from an 'initial' negative infinite time. On the other hand, $h(x)=a|x|(a>0)$ is a stationary weak solution of (11) for $m>2$ (see Remark 1.2); hence our results yield a special mechanism of non-uniqueness for solutions of (1).

The non-uniqueness of weak solutions for $m \in(0,5)$ has been shown by Beretta, Bertsch and Dal Passo in [3]. In order to show this, the authors regularize (1) and this allows to construct solutions which stay positive, while other solutions for the same initial data vanish at some specific points in space. The self-similar solutions obtained in the current paper yield a mechanism on how this non-uniqueness may take place at isolated touch-down points (we refer also to the discussion at the end of Section 2). For existence theory of weak solutions of (1), we refer to, e.g., [3, 4, 17] in the one-dimensional case, and to [9, 12, 23. in the case of higher space dimensions. Corresponding results on existence and uniqueness of classical solutions have been addressed in, e.g., [16, 18, 19, 25, 26, see also [21]. A well-posedness result for $m=1$ in the case of partial wetting is included in [27, 28].

Most of the analysis on self-similar solutions of (11) has focused on the study of source-type solutions. In, e.g., [6], it has been shown that source-type selfsimilar solutions exist for all $m \in(0,3)$, but not for $m=3$; for a corresponding result in higher space dimensions, we refer to [15. These results are consistent with the conjecture that droplet spreading is not possible for $m \geq 3$ (see [14, 24]). Additional regularity up to the moving boundary of the source-type self-similar solutions with $m \in\left(\frac{3}{2}, 3\right)$ is studied in [17. A source-type self-similar solution 
with zero-contact angle and where a portion of the boundary may undergo drainage is studied in [5] for $m \in(0,3)$.

Structure of the paper. In Section 2 we state our main results on the existence of solutions which exhibit self-similar lifting. The proof of the theorem is given in Section 3. In the Appendix, we formally derive the behavior of the profile for two identical droplets merging in a self-similar way.

\section{Statement of the results}

We consider initial data with an isolated touch-down point at the origin, i.e.

$$
h_{\text {in }}(0)=0 \quad \text { and } \quad h_{\text {in }}(x)>0 \quad \text { for } x \neq 0 .
$$

Moreover, we assume that the initial data is symmetric around this point, $h_{\text {in }}(-x)=h_{\text {in }}(x)$, with non-zero slope on both sides, i.e.

$$
\lim _{x \rightarrow 0}\left|h_{\mathrm{in}, \mathrm{x}}(x)\right|=a \quad \text { for some } \quad a>0 .
$$

We consider the existence of a self-similar solution of the form

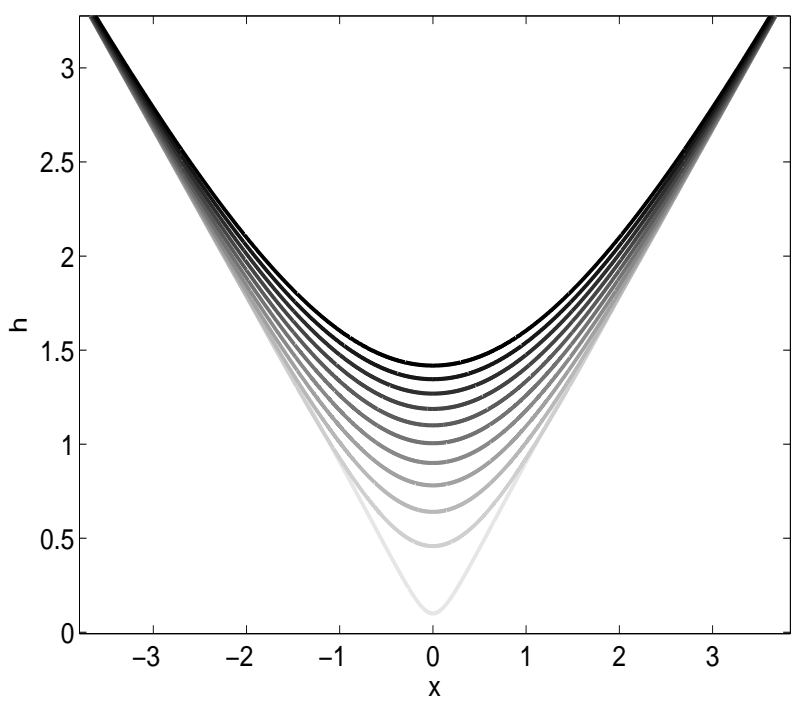

Figure 1: The picture shows solutions $h(x, t)=t^{\alpha} f\left(|x| / t^{\alpha}\right)$, where $f$ satisfies (10)-(12) with $m=2$ and for $t \in[0.01,1.01)$ at increments of size 0.2 . The solution is calculated using the ODE solver ode45 of MATLAB (a fourth order Runge-Kutta algorithm) by iterating in the shooting parameter $\kappa$.

$$
h(x, t)=t^{\frac{1}{4-m}} f(y), \quad y=\frac{|x|}{t^{\frac{1}{4-m}}} \quad \text { if } m \in(0,4)
$$


for $x \in \mathbb{R}$ and for some $f:[0, \infty) \rightarrow \mathbb{R}$. In order to preserve the linear behavior of solutions in the outer region away from the touch-down point, we require (see below) that $f$ grows linearly as $|y| \rightarrow \infty$. For $m=4$, we also consider solutions of the form:

$$
h(x, t)=e^{t b} f(y), \quad y=\frac{|x|}{e^{t b}}
$$

where $b>0$ is a free parameter. For $m \in(0,4)$, the solution is defined for $t>0$, while the problem is formulated for $t \in \mathbb{R}$ when $m=4$. With this ansatz and defining the parameter

$$
\alpha:=\left\{\begin{array}{cl}
\frac{1}{4-m} & \text { if } m \in(0,4), \\
b & \text { if } m=4,
\end{array}\right.
$$

the equation (11) becomes the fourth order ODE

$$
\alpha\left(f-y f_{y}\right)+\left(f^{m} f_{y y y}\right)_{y}=0 \quad \text { for } y \in(0, \infty) .
$$

By our assumption that the solution is even, we have $f_{y}(0)=f_{y y y}(0)=0$, which leaves the two free parameters $f(0)$ and $f_{y y}(0)$. In view of the scaling invariance $(y, f) \mapsto\left(\lambda y, \lambda^{\frac{4}{m}} f\right)$ for any $\lambda>0$ of (10), it is enough to consider the initial conditions $f(0)=1, f_{y y}(0)=\kappa$ for some $\kappa \in \mathbb{R}$. Summarizing, we look for a solution of (10) which satisfies the initial conditions

$$
f(0)=1, \quad f_{y}(0)=0, \quad f_{y y}(0)=\kappa>0, \quad f_{y y y}(0)=0,
$$

and which satisfies the behavior

$$
\frac{f(y)}{y} \rightarrow a>0 \quad \text { as } y \rightarrow \infty,
$$

for some $a>0$ which is not prescribed a priori.

Our main result states the existence of a solution for the problem (10)-12) for $m \in(0,4]$ :

Theorem 2.1 (Existence of a self-similar solution).

(i) Let $m \in(0,4)$. Then there exists $\kappa \in(0, \sqrt{12 \alpha})$ and $a>0$ such that the problem (10) -12) has a solution.

(ii) Let $m=4$ and let $b>0$. Then there exists $\kappa \in(0, \sqrt{12 \alpha})$ and $a>0$ such that the problem (10) - (12) has a solution that satisfies

$$
f(y)=a y+\operatorname{Re}\left[K y^{1+z_{0}}\right]+o\left(y^{1+\operatorname{Re}\left(z_{0}\right)}\right) \quad \text { as } y \rightarrow \infty
$$

for $z_{0}, K \in \mathbb{C}$ with $\operatorname{Re}\left(z_{0}\right)<-1$. Here, $z_{0}$ is the root of $\left(1-z^{2}\right)(2+z)+\frac{b}{a^{4}}$ with the largest negative real part and, for definiteness, with $\operatorname{Im}\left(z_{0}\right) \geq 0$. 
Using the scaling invariance of the equation, for $m \in(0,4)$ we obtain a solution $f$ of (10)-(12) for any prescribed slope $a$ at infinity (the corresponding solution does not necessarily satisfy the condition $f(0)=1$ ). For $m=4$, the same type of rescaling leaves the slope at infinity invariant. However, by rescaling in $y$ but not in $f$ (preserving the condition $f(0)=1$ ) we obtain a solution of the rescaled equation for some $b>0$, with the desired slope at infinity. As a consequence of Theorem 2.1 the thin-film equation (1) with initial data $h_{\text {in }}(x)=a|x|$ admits a solution which exhibits self-similar lifting for any prescribed slope at infinity:

Corollary 2.2 (Self-similar lifting for the thin-film equation).

(i) Let $m \in(0,4)$. Then, for any $a>0$, there exists a weak solution $h$ of (1D) with initial data $h_{\mathrm{in}}(x)=a|x|$ of the form (7).

(ii) Let $m=4$. Then, for any $a>0$, there exists $b>0$ and a self-similar weak solution $h$ of (11) of the form (7), defined for all $t \in \mathbb{R}$, and such that

$$
h(x, t)=a|x|+\operatorname{Re}\left[K\left(\frac{|x|}{e^{b t}}\right)^{1+z_{0}}\right]+o\left(\left(\frac{|x|}{e^{b t}}\right)^{1+\operatorname{Re}\left(z_{0}\right)}\right)
$$

uniformly as $t \rightarrow-\infty$ for $x \in[-R, R]$ for any fixed $R>0$. Here, $K, z_{0} \in$ $\mathbb{C}$ are the same constants as in Theorem 2.1.

It is an open question whether the self-similar solution in Theorem 2.1 is unique.

For $m \in(0,4)$, the solution in Corollary 2.2 describes lifting at time $t=0$. For $m=4$, the solution is defined for all $t \in \mathbb{R}$ and describes lifting in infinite time, in the sense that the cone $h(x)=a|x|$ is approached in the limit $t \rightarrow-\infty$. Note that the corresponding solutions do not represent lifting if $m>4$, but instead, such solutions would convergence to the cone as $t \rightarrow \infty$. In fact, we do not expect that lifting is possible in this case. It is an open question whether the self-similar solution in Theorem 2.1 is unique.

Figure 1 illustrates the evolution given by the self-similar solutions for the case $m=2$.

Finally, we recall that the self-similar solutions obtained in this paper can be understood as locally describing the merging of two adjacent droplets. In the Appendix, we present the formal asymptotics of two initially stationary identical droplet that meet at an isolated touch-down point. The construction of these solutions should follow from a combination of the arguments in this paper with a suitable localization argument.

Strategy of the proof. The proof of Theorem 2.1 is based on a shooting argument with shooting parameter $\kappa=f_{y y}(0)$. We first identify two invariant regions $\Sigma_{ \pm}$. We show that any solution which enters the invariant $\Sigma_{-}$region (related to small $\kappa$ ) exhibits touch-down to zero at some finite $y^{*}>0$, i.e.

$$
f\left(y^{*}\right)=0 \quad \text { and } \quad f(y)>0 \text { for all } y \in\left(0, y^{*}\right)
$$


for some $y^{*} \in(0, \infty)$. On the other hand, solutions which enter the invariant region $\Sigma_{+}($related to large $\kappa$ ) exhibit at least quadratic growth, i.e.

$$
f(y) \geq c y^{2} \quad \text { for all } y \in(0, \infty)
$$

and for some $c>0$. We then invoke the energy formula

$$
\frac{d}{d y}\left(\alpha\left(f f_{y}-\frac{1}{2} y\left(f_{y}\right)^{2}\right)+f^{m} f_{y y} f_{y y y}\right)=\frac{\alpha}{2} f_{y}^{2}+f^{m} f_{y y y}^{2},
$$

(obtained by multiplying (10) with $f_{y y}$ ) and another energy identity (see Lemma 3.4) to show that there are solutions that fall into neither of these invariant regions. The proof is then concluded by showing that these solutions necessarily satisfy the linear growth (12).

Discussion: The self-similar solutions found in the current work give a specific mechanism of non-uniqueness related to topological changes of the positivity set of the solution. Namely, we show that if $m \in(2,4)$ there are at least two weak solutions, one that is the stationary solution $h_{\text {in }}(x)=a|x|$ and another solution exhibiting self-similar lifting. We note that, since the initial condition for the self-similar solution represent stationary solutions, the lifting can in principle occur at any time, thus giving an infinite set of solutions with the same initial data. We believe that for other initial data with, e.g., $h_{\text {in }}(x) \approx|x|^{\beta}$ for small $|x|$, other type of self-similar lifting solutions may also exist.

For weak solutions in the sense of Definition 1.1 with initial data $h_{\text {in }}(x)=$ $a|x|$, our result suggests the following picture:

(i) If $m \in(0,2]$, there exists a unique weak (self-similar) solution that lifts up.

(ii) If $m \in(2,4)$, there exists a stationary solution as well as infinitely many solutions which exhibit lifting at arbitrary time.

(iii) If $m \geq 4$, there exists a unique weak solution that is $h_{\text {in }}(x)=a|x|$.

The statements (i) and (iii) are conjectures while (ii) follows from Corollary 2.2 While the stationary solutions $h(x)=a|x|$ for $m \in(2,4)$ are weak solutions with zero dissipation, our self-similar solutions have finite positive dissipation (see Corollary 3.11). This raises the natural question as to whether the criterion of maximum dissipation yields uniqueness of weak solutions.

It is now pertinent to compare our results with the non-uniqueness result of weak solutions by Beretta, Bertsch and Dal Passo. More precisely, in [3], the authors show non-uniqueness for weak solutions of (1) on a finite interval for $m \in(0,5)$. They show the existence of entropy solutions for $m \in(0,5)$ which converge to their mean as $t \rightarrow \infty$ and become strictly positive in finite time for non-trivial initial data. Furthermore, they show that for any $m>0$ there exist weak solutions satisfying (2)-(4), there are ones that preserve touchdown points in space and time if $m \in(0,3)$, and others that have fixed compact 
support in space and time if $m \geq 3$. As a consequence, weak solutions with finite dissipation, i.e. solutions satisfying (4) and (2), are not unique if $m \in(0,5)$. In particular, Theorem 3.2 in [3] implies that for $m \geq 3$ there exists a positive solution if the initial data $h_{\text {in }}$ is sufficiently large near the touch-down points. For initial data of the form $|x|^{\beta}$, the condition is satisfied if $\beta \in\left(0, \frac{1}{m-3}\right)$ for $m \neq 3$ (and arbitrary $\beta$ if $m=3$ ). It would be interesting to show whether lifting can be realized by self-similar lifting with such initial data. On the other hand, uniqueness of entropy solutions is still an open question (see also [10, Theorem 5.1 and 5.2]).

\section{Proof of the results}

\subsection{Reformulation of the problem and invariant regions}

We write (10) as a system of four first order equations, where the new variables are adapted to the scaling invariance of (10). Namely, we introduce

$$
\Phi:=\frac{f}{y^{\frac{4}{m}}}, \quad W:=\frac{y f_{y}}{f}, \quad Q:=y^{\frac{2}{3}} f^{\frac{m-3}{3}} f_{y y}, \quad Z:=y^{\frac{1}{3}} f^{\frac{2 m-3}{3}} f_{y y y},
$$

and the independent variable $\xi \in \mathbb{R}$ by

$$
y=e^{\xi} .
$$

In terms of $(\Phi, W, Q, Z)$ and $\xi$, (10) turns into the following system of four first order ordinary differential equations:

$$
\left\{\begin{array}{l}
\frac{d \Phi}{d \xi}=\Phi\left(W-\frac{4}{m}\right) \\
\frac{d W}{d \xi}=\frac{Q}{\Phi^{\frac{m}{3}}}+W(1-W) \\
\frac{d Q}{d \xi}=\left(\frac{2}{3}+\frac{m-3}{3} W\right) Q+\frac{Z}{\Phi^{\frac{m}{3}}} \\
\frac{d Z}{d \xi}=\alpha \frac{W-1}{\Phi^{\frac{m}{3}}}+\left(\frac{1}{3}-\frac{m+3}{3} W\right) Z
\end{array}\right.
$$

We use the compact notation $X=(\Phi, W, Q, Z)$ to denote any point in the phasespace associated to the dynamical system (19). We shall also write $X(\cdot ; \kappa)$ as the solution of (19) that corresponds to the solution of (10)-(11) when we want to explicitly indicate the dependence on the value of $\kappa$.

We find the following invariant regions of (19):

Lemma 3.1 (The invariant regions $\Sigma_{ \pm}$).

(i) The domains

$$
\Sigma_{ \pm}:=\left\{(\Phi, W, Q, Z) \in \mathbb{R}^{4}: \Phi>0, \pm(W-1)>0, \pm Q>0, \pm Z>0\right\}
$$

are invariant regions for the dynamical system (19). 
(ii) If $X(\xi) \in \Sigma_{+}$for some $\xi \in \mathbb{R}$, then the solution exhibits the behavior (15).

(iii) If $X(\xi) \in \Sigma_{-}$for some $\xi \in \mathbb{R}$, then the solution exhibits the behavior (14).

Proof. (i): Suppose that $X\left(\xi_{0}\right) \in \Sigma_{+}$for some $\xi_{0} \in \mathbb{R}$. We first note that by the first equation in (19), the condition $\Phi>0$ is preserved for $\xi>\xi_{0}$. From the second equation in (19) and since $Q>0$, we get $W_{\xi} \geq W(1-W)$ which implies that the property $W>1$ is preserved as long as $Q>0$. From the third equation in (19) and since $Z>0$, we get that the property $Q>0$ is preserved as long as $Z>0$. From the fourth equation in (19) and since $W>1$, we get $Z_{\xi} \geq\left(\frac{1}{3}-\left(1+\frac{m}{3}\right) W\right) Z$ which implies that the property $Z>0$ is preserved as long as $W>1$. This shows that the region $\Sigma_{+}$is invariant, i.e. $X(\xi) \in \Sigma_{+}$for all $\xi \geq \xi_{0}$. The argument for the invariance of $\Sigma_{-}$proceeds analogously.

(ii): Suppose that $X \in \Sigma_{+}$for all $\xi>\xi_{0}$ and some $\xi_{0} \in \mathbb{R}$. Since $Q>0$ and $Z>0$, we have $f_{y y y}>0$ and $f_{y y}>0$ by the definition of $Z$ and $Q$, and hence $f_{y y}(y)>f_{y y}\left(y_{0}\right)>0$ for all $y \geq y_{0}=e^{\xi_{0}}$. In particular, $f \geq C y^{2}$ for all $y \geq y_{0}$ for some $C>0$ which depends on $y_{0}$. Since $f>0$ and $f$ is continuous in $\left(0, y_{0}\right)$, we obtain (15).

(iii): Suppose that $X \in \Sigma_{-}$for all $\xi \geq \xi_{0}$ and some $\xi_{0} \in \mathbb{R}$. Using the first equation in (19), we calculate

$$
\begin{array}{r}
\Phi^{\frac{m}{3}}(\xi) \stackrel{\text { (19) }}{=}\left(\Phi\left(\xi_{0}\right) \exp \left(\int_{\xi_{0}}^{\xi}\left(W(s)-\frac{4}{m}\right) d s\right)\right)^{\frac{m}{3}} \\
=C \exp \left(\int_{\xi_{0}}^{\xi}\left(\frac{m}{3} W(s)-\frac{4}{3}\right) d s\right) .
\end{array}
$$

Since $Z<0$, from the third equation in (19) we obtain

$$
Q(\xi)<-C \exp \left(\int_{\xi_{0}}^{\xi}\left(\frac{2}{3}+\frac{m-3}{3} W(s)\right) d s\right)<0 \quad \text { for } \xi \geq \xi_{0} .
$$

Using the second equation in (19) together with (20) and (22), we obtain

$$
\frac{d W(\xi)}{d \xi}<-C \exp \left(\int_{\xi_{0}}^{\xi}(2-W(s)) d s\right)+W(\xi)(1-W(\xi)) \quad \text { for } \xi \geq \xi_{0} .
$$

Since $W<1$, this implies $W_{\xi} \leq-C e^{\xi}$ for $\xi$ sufficiently large. It follows that $W<0$ eventually and hence $f_{y}<0$ for sufficiently large $y=e^{\xi}$. Since $Q<0$ and hence $f_{y y}<0$, it follows that there is $y^{*}>0$ with $f\left(y^{*}\right)=0$.

The next lemma relates the value of $\kappa$ to the invariant regions $\Sigma_{ \pm}$:

Lemma 3.2 (The case of large and small $\kappa$ ).

(i) If $\kappa<0$, then $X(\cdot ; \kappa)$ enters $\Sigma_{-}$, i.e. there is $\xi_{0} \in \mathbb{R}$ with $X\left(\xi_{0} ; \kappa\right) \in \Sigma_{-}$.

(ii) If $\kappa>\sqrt{12 \alpha}$, then $X(\cdot ; \kappa)$ enters $\Sigma_{+}$, i.e. there is $\xi_{0} \in \mathbb{R}$ with $X\left(\xi_{0} ; \kappa\right) \in$ $\Sigma_{+}$. 
Proof. (i): If $\kappa<0$, (10) and (11) imply that for a small enough $y_{0}=e^{\xi_{0}}>0$ we have $W\left(\xi_{0}\right)<0, Q\left(\xi_{0}\right)<0$ and $Z\left(\xi_{0}\right)<0$ and hence $X\left(\xi_{0} ; \kappa\right) \in \Sigma_{-}$.

(ii): By integrating (10) once, we get $f^{m}(y) f_{y y y}(y)=\alpha \int_{0}^{y}\left(s f_{s}(s)-f(s)\right) d s$. Since $\left(y f_{y}-f\right)_{y}=y f_{y y}$ and $\left(y f_{y}-f\right)(0)=-1$, we obtain

$$
f_{y y y}(y)=\frac{\alpha}{f^{m}(y)}\left(\int_{0}^{y} \int_{0}^{s} r f_{r r}(r) d r d s-y\right) .
$$

Let $\bar{y} \in(0, \infty]$ be the largest value such that the solution is defined and $f_{y y}(y)>$ $\frac{\kappa}{2}$ for all $y \in I:=(0, \bar{y})$. For $y \in I$, we have $f_{y}(y)>0$ and $f(y)>1$. By (23)

we also have $f_{y y y}(y)>-\alpha y$ and hence $f_{y y}(y) \geq \kappa-\alpha \frac{y^{2}}{2}$ for $y \in I$. In turn, this implies $y^{*}:=\sqrt{\kappa / \alpha} \in I$. We thus have $f_{y y}(y) \geq \frac{\kappa}{2}$ for all $y<y^{*}$ and hence

$$
f_{y y y}\left(y^{*}\right) \stackrel{\sqrt[23]{2}}{\geq} \frac{\alpha y^{*}}{f^{m}\left(y^{*}\right)}\left(\frac{\kappa^{2}}{12 \alpha}-1\right)>0 \quad \text { if } \quad \kappa>\sqrt{12 \alpha} .
$$

This implies $Z\left(\xi^{*}\right)>0$ where $y^{*}=e^{\xi^{*}}$. Clearly, we have $f_{y y}\left(y^{*}\right)>0$ and hence $Q\left(\xi^{*}\right)>0$. We also calculate

$$
y^{*} f_{y}\left(y^{*}\right)-f\left(y^{*}\right)=\int_{0}^{y^{*}} s f_{s s} d s-1 \geq \frac{\kappa^{2}}{4 \alpha}-1>0 \quad \text { if } \kappa>\sqrt{12 \alpha},
$$

which implies $W\left(\xi^{*}\right)>1$ by the definition (17) of $W$. The above calculations hence yield $X\left(\xi^{*} ; \kappa\right) \in \Sigma_{+}$if $\kappa>\sqrt{12 \alpha}$.

We can now conclude that there exist values of $\kappa$ such that the corresponding solutions to (10)-(11) enter neither of the invariant regions:

Lemma 3.3 (Shooting argument). Let

$$
I_{ \pm}:=\left\{\kappa \in \mathbb{R}: X(\xi ; \kappa) \in \Sigma_{ \pm} \text {for some } \xi>0\right\}, \quad I_{0}:=\mathbb{R} \backslash\left(I_{+} \cup I_{-}\right) .
$$

Then, the sets $I_{ \pm} \subset \mathbb{R}$ are open, nonempty and disjoint and, hence, $I_{0} \neq \emptyset$.

Proof. By definition, the sets $\Sigma_{ \pm}$are open. By the continuous dependence of solutions on the initial data, the sets $I_{ \pm}$are hence also open. By Lemma 3.1 the sets $I_{ \pm}$are disjoint. By Lemma 3.2 the sets $I_{ \pm}$are non-empty.

In order to conclude the proof of Theorem 2.1 we have to characterize the behavior as $\xi \rightarrow \infty$ of the solutions with initial condition in $I_{0}$ and show that this corresponds to (12). We do this in the next two sections.

\subsection{Energy formulas and corollaries}

The following two energy formulas are essential for the proof of Theorem 2.1] 
Lemma 3.4 (Energy Formula I). We define $E_{1}(y)$ by

$$
E_{1}(y):=\alpha\left(f f_{y}-\frac{1}{2} y f_{y}^{2}\right)+f^{m} f_{y y} f_{y y y} .
$$

Then, if $f$ is a smooth solution of (10), $E_{1}$ is an increasing quantity with

$$
\frac{d}{d y} E_{1}(y)=\frac{\alpha}{2} f_{y}^{2}+f^{m} f_{y y y}^{2}
$$

In particular, if $f$ solves (10)-(11), then $E_{1}(0)=0$ and $E_{1}(y)>0$ for all $y>0$.

Proof. Multiplying (10) by $f_{y y}$ we get

$$
\begin{aligned}
0 & =\alpha\left(f f_{y y}-y f_{y} f_{y y}\right)+\left(f^{m} f_{y y y}\right)_{y} f_{y y} \\
& =\left(\alpha\left(f f_{y}-\frac{1}{2} y f_{y}^{2}\right)+f^{m} f_{y y y} f_{y y}\right)_{y}+\alpha\left(-f_{y}^{2}+\frac{1}{2} f_{y}^{2}\right)-f^{m} f_{y y y}^{2},
\end{aligned}
$$

and rearranging terms we obtain the result.

Lemma 3.5 (Energy Formula II). We define $E_{2}(y)$ by

$$
E_{2}(y):=-\frac{\alpha}{2 y}\left(f-y f_{y}\right)^{2}+f^{m} f_{y y} f_{y y y} .
$$

Then, if $f$ is a smooth solution of (10), $E_{2}$ is an increasing quantity with

$$
\frac{d}{d y} E_{2}(y)=\frac{\alpha}{2 y^{2}}\left(f-y f_{y}\right)^{2}+f^{m} f_{y y y}^{2} .
$$

In particular, if $f$ is a solution of (10)-(11) then $\lim _{y \rightarrow 0^{+}} E_{2}(y)=-\infty$.

Proof. We start from the energy formula of Lemma 3.4. We calculate

$$
\alpha \frac{d}{d y}\left(-\frac{f^{2}}{2 y}\right)=\alpha\left(-\frac{f f_{y}}{y}+\frac{f^{2}}{2 y^{2}}\right) .
$$

We then take the sum of this identity with the first energy equality (26),

$$
\frac{d}{d y}\left(\alpha\left(f f_{y}-\frac{1}{2} y f_{y}^{2}\right)+f^{m} f_{y y} f_{y y y}\right)=\frac{\alpha}{2} f_{y}^{2}+f^{m} f_{y y y}^{2} .
$$

Taking the sum completes the squares in both sides of the resulting equation and thus yields the assertion of the lemma.

Remark 3.6. In the variables (17) and (18), the energy formula for $E_{1}$ of Lemma 3.4 becomes

$$
\frac{d}{d \xi}\left(e^{\frac{8-m}{m} \xi} \Phi^{2}\left(-\frac{\alpha}{2} W(W-2)+Q Z\right)\right)=e^{\frac{8-m}{m} \xi} \Phi^{2}\left(\frac{\alpha}{2} W^{2}+\frac{Z^{2}}{\Phi^{\frac{3}{m}}}\right)
$$

and the energy formula for $E_{2}$ of Lemma 3.5 takes the form

$$
\frac{d}{d \xi}\left(e^{\frac{8-m}{m} \xi} \Phi^{2}\left(-\frac{\alpha}{2}(W-1)^{2}+Q Z\right)\right)=e^{\frac{8-m}{m} \xi} \Phi^{2}\left(\frac{\alpha}{2}(W-1)^{2}+\frac{Z^{2}}{\Phi^{\frac{3}{m}}}\right) .
$$


With the aid of these energies we now show the following lemma:

Lemma 3.7. Let $\kappa \geq 0$ and $X(\cdot ; \kappa)=(\Phi, W, Q, Z)$ be the corresponding solution of (19). Then:

(i) If $W\left(\xi^{*}\right)<0$ for some $\xi^{*} \in \mathbb{R}$, then $X\left(\xi^{*} ; \kappa\right) \in \Sigma_{-}$.

(ii) If $W\left(\xi_{*}\right)>2$ for some $\xi^{*} \in \mathbb{R}$, then $X\left(\xi^{*} ; \kappa\right) \in \Sigma_{+}$.

Proof. If $\kappa=0$, then the solution satisfies $W(\xi)=Q(\xi)=Z(\xi)=0$ for all $\xi \in \mathbb{R}$ and there is nothing to prove. Hence, in the following we assume that $\kappa>0$. By the initial conditions, we have $W(\xi) \rightarrow 0$ as $\xi \rightarrow-\infty$ and since $\kappa>0$, we also have $W(\xi) \in(0,2)$ for $\xi$ sufficiently small.

(i): Suppose that $W\left(\xi_{*}\right)<0$ for some $\xi_{*} \in \mathbb{R}$ and let

$$
\bar{\xi}=\min \{\xi \in \mathbb{R}: W(\xi)=0\} .
$$

Since $W \in(0,2)$ for $\xi$ sufficiently small and since $W$ is continuous, the minimum exists and we have $-\infty<\bar{\xi}<\xi_{*}<\infty$. By the definition of $\bar{\xi}$, we have $W_{\xi}(\bar{\xi}) \leq 0$. On the other hand, by the second equation in (19), we have $W_{\xi}(\xi)=$ $Q(\bar{\xi}) / \Phi^{\frac{m}{3}}(\bar{\xi})$ and hence $Q(\bar{\xi}) \leq 0$. By Lemma 3.4 we have $E_{1}(y)>0$ for all $y>0$. In view of Remark 3.6. this implies

$$
-\frac{\alpha}{2} W(\bar{\xi})(W(\bar{\xi})-2)+(Q Z)(\bar{\xi})=(Q Z)(\bar{\xi})>0
$$

Since $Q(\bar{\xi}) \leq 0$, this yields $Q(\bar{\xi})<0$ and $Z(\bar{\xi})<0$. This finally implies that $X(\bar{\xi} ; \kappa) \in \Sigma_{-}$and Lemma 3.1 implies the assertion.

(ii): Suppose now that $W\left(\xi_{*}\right)>2$ for some $\xi_{*} \in \mathbb{R}$. We define

$$
\bar{\xi}=\min \{\xi \in \mathbb{R}: W(\xi)=2\} .
$$

Since $W \in(0,2)$ for $\xi$ sufficiently small and since $W$ is continuous, the minimum exists and we have $-\infty<\bar{\xi}<\xi_{*}<\infty$. By the second equation in (19) we obtain $Q(\bar{\xi}) \geq 0$. Arguing as before, we also deduce that $(Q Z)(\bar{\xi})>0$, thus we get $Q(\bar{\xi})>0$ and $Z(\bar{\xi})>0$. This implies $X(\bar{\xi} ; \kappa) \in \Sigma_{+}$and (ii) follows.

Solutions exhibiting touch-down in finite time enter the invariant region $\Sigma_{-}$:

Lemma 3.8. Let $\kappa \in \mathbb{R}$ and let $X(\cdot ; \kappa)=(\Phi, W, Q, Z)$ be the corresponding solution for the dynamical system (19). If the maximal time of existence $\xi_{M}$ is finite, then, there exists $\bar{\xi}<\xi_{M}$ with $X(\bar{\xi} ; \kappa) \in \Sigma_{-}$.

Proof. If $\kappa<0$ or $\kappa \geq 0$ and $W(\xi)<0$ for some $\xi \in\left(0, \xi_{M}\right)$, then by Lemma 3.2 and Lemma 3.7. the solution enters $\Sigma_{-}$and there is nothing to prove. We hence assume that $\kappa \geq 0$ and $W(\xi) \geq 0$ for all $\xi \in\left[0, \xi_{M}\right)$. Then the first equation in (19) yields $\Phi(\xi) \geq c_{0} e^{-\frac{4 \xi}{m}}$ as long as the solution is defined. By standard ODE theory, this implies that the solutions of (19) are defined globally, which gives a contradiction. 


\subsection{Behavior for large $\xi$}

In this section, we consider the asymptotic behavior of solutions which enter neither of the two invariant regions $\Sigma_{ \pm}$(i.e. $\kappa \in I_{0}$ ). We first show that these solutions are globally defined and satisfy certain bounds in terms of $\Phi, W$ :

Lemma 3.9. Let $\kappa \in I_{0}$ and let $X(\cdot ; \kappa)=(\Phi, W, Q, Z)$ be the corresponding solution of the dynamical system (19). Then $X(\cdot, \kappa)$ is globally defined with

$$
\lim _{\xi \rightarrow \infty} \Phi(\xi)=K \quad \text { where } \begin{cases}K=0 & \text { if } m \in(0,4) \\ K \geq 0 & \text { if } m=4 .\end{cases}
$$

Furthermore, $W$ satisfies

$$
0 \leq W(\xi) \leq 2 \quad \text { for all } \xi \in \mathbb{R} .
$$

Proof. By Lemma 3.8, the solution $X(\cdot ; \kappa)$ is defined for all $\xi \in \mathbb{R}$. By Lemma 3.7 (36) holds. It hence remains to show (35). By Lemma 3.5 $E_{2}$ is increasing. Hence, one of the following two cases holds: Either we have $E_{2}(y) \leq 0$ for all $y>0$ or there exists $y^{*}$ such that $E_{2}(y)>0$ for all $y>y^{*}$. We consider these two cases separately:

Case 1: In this case, we assume that $E_{2}(y) \leq 0$ for all $y>0$. Integrating (28) in the interval $(1, \infty)$ we then obtain

$$
\int_{1}^{\infty}\left(\frac{\alpha}{2}\left(f_{s}-\frac{f}{s}\right)^{2}+f^{m} f_{s s s}^{2}\right) d s=-E_{2}(1)<\infty,
$$

since $E_{2}(y)$ cannot blow up at a finite $y$ (it is increasing and non-positive). In particular, we get

$$
\int_{1}^{\infty}\left|f_{s}-\frac{f}{s}\right| \frac{d s}{s} \leq\left(\int_{1}^{\infty}\left|f_{s}-\frac{f}{s}\right|^{2} d s\right)^{\frac{1}{2}}\left(\int_{1}^{\infty} \frac{d s}{s^{2}}\right)^{\frac{1}{2}}<\infty .
$$

Using the observation that $\left(\frac{f}{y}\right)_{y}=\frac{1}{y}\left(f_{y}-\frac{f}{y}\right)$, we compute

$$
\frac{f(y)}{y}=f(1)+\int_{1}^{y}\left(f_{s}-\frac{f}{s}\right) \frac{d s}{s} .
$$

In view of (38), the right hand side of (39) is absolutely convergent as $y \rightarrow \infty$ and the limit is finite. Therefore, also the limit $K:=\lim _{y \rightarrow \infty} \frac{f(y)}{y} \geq 0$ exists and is finite. Since $\Phi=f / y^{\frac{4}{m}}$, in particular we get $\Phi(\xi) \rightarrow 0$ as $\xi \rightarrow \infty$ if $m \in(0,4)$.

Case 2: In this case, we assume that there exists $y^{*}>0$ such that $E_{2}(y)>0$ for all $y>y^{*}$. In view of (31), we then have $(Q Z)(\xi)>0$ for all $\xi>\xi^{*}$ where $y^{*}=e^{\xi^{*}}$. In the following, we assume $\xi>\xi^{*}$. By Lemma 3.7. we also have $0 \leq W(\xi) \leq 2$. We claim that

$$
\frac{d W}{d \xi}<0 \quad \text { if } W(\xi)>1 \quad \text { and } \quad \frac{d W}{d \xi}>0 \quad \text { if } W(\xi)<1 .
$$


Indeed, if $W(\xi)>1$ and $W_{\xi}(\xi) \geq 0$, then by the second equation in (19) we get $Q(\xi)>0$. Since $(Q Z)(\xi)>0$, this implies $Z(\xi)>0$ and hence $X(\xi ; \kappa) \in \Sigma_{+}$ which contradicts the assumption that $\kappa \in I_{0}$. Similarly, if $W(\xi)<1$ and $W_{\xi}(\xi) \leq 0$, then by the second equation in (19) we have $Q(\xi)<0$. Since $(Q Z)(\xi)>0$, this implies $Z(\xi)<0$ and hence $X(\xi ; \kappa) \in \Sigma_{-}$which, again, contradicts our assumptions. Therefore (40) holds. It follows that either $0 \leq$ $W(\xi) \leq 1$ and $W$ is non decreasing for all $\xi \geq \xi^{*}$, or that $1 \leq W(\xi) \leq 2$ and $W$ is non increasing for all $\xi \geq \xi^{*}$. Therefore, there is $L \in[0,2]$ such that $\lim _{\xi \rightarrow \infty} W(\xi)=L$.

We next show that $L=1$. Arguing by contradiction, we assume that $L \neq 1$. If $L \in(1,2]$, then as before and by (40), we have $W_{\xi}(\xi)<0$ and $Q(\xi)<0$ for all $\xi>\xi^{*}$. Analogously, if $L \in[0,1)$, then $W_{\xi}(\xi)>0$ and $Q(\xi)>0$ for all $\xi>\xi^{*}$. In both cases, by the second equation in (19), we get $\left|W_{\xi}\right| \geq W|W-1|$ and

$$
\int_{\xi^{*}}^{\infty} W(\xi)|W(\xi)-1| d \xi \leq \int_{\xi^{*}}^{\infty}\left|\frac{d W(\xi)}{d \xi}\right| d \xi=\left|W\left(\xi^{*}\right)-L\right|<\infty
$$

which implies $L=1$ (the case $L=0$ contradicts the dynamics of (19)).

Since $W(\xi) \rightarrow 1$ as $\xi \rightarrow \infty$ and by the first equation in (19), we immediately get $\Phi(\xi) \rightarrow 0$ as $\xi \rightarrow \infty$ if $m \in(0,4)$. If $m=4$, by integrating the first equation in (19), we get for $\xi_{2} \geq \xi_{1} \geq \xi^{*}$,

$\left|\ln \Phi\left(\xi_{1}\right)-\ln \Phi\left(\xi_{2}\right)\right|=\int_{\xi_{1}}^{\xi_{2}}(W(\xi)-1) d \xi \leq \frac{2}{W\left(\xi_{1}\right)} \int_{\xi_{1}}^{\infty} W(\xi)|W(\xi)-1| d \xi \stackrel{\text { 沎 }}{\longrightarrow} 0$

as $\xi_{1} \rightarrow \infty$. We have used that $W(\xi) \geq 2 W\left(\xi_{1}\right)$ if $W\left(\xi_{1}\right) \geq 1$ and that $W(\xi)$ increases monotonically if $W\left(\xi_{1}\right)<1$. This implies $\ln \Phi(\xi) \rightarrow \ln K<\infty$ for some $K>0$ and hence $\Phi(\xi) \rightarrow K>0$ as $\xi \rightarrow \infty$.

The asymptotic behavior for solutions which enter neither of the two invariant regions $\Sigma_{ \pm}$is described in the next proposition:

Proposition 3.10. Suppose that $\kappa \in I_{0}$ and let $X(\cdot ; \kappa)=(\Phi, W, Q, Z)$ be the corresponding solution for the dynamical system (19).

(i) If $m \in(0,4)$, then we have

$$
\lim _{\xi \rightarrow \infty} \Phi(\xi)=0, \lim _{\xi \rightarrow \infty} W(\xi)=1, \lim _{\xi \rightarrow \infty} Q(\xi)=0, \lim _{\xi \rightarrow \infty} Z(\xi)=0 .
$$

More precisely, for some $a>0$ and for $K_{0}=\frac{3}{8}(4-m)^{\frac{4}{3}} a^{\frac{m}{3}}$ we have

$$
\lim _{\xi \rightarrow \infty} e^{\frac{4-m}{m} \xi} \Phi(\xi)=a>0, \lim _{\xi \rightarrow \infty} e^{K_{0} e^{\frac{4-m}{3} \xi}}\|(W-1, Q, Z)\|<\infty .
$$

(ii) If $m=4$, then we have

$$
\lim _{\xi \rightarrow \infty} \Phi(\xi)=a>0, \lim _{\xi \rightarrow \infty} W(\xi)=1, \lim _{\xi \rightarrow \infty} Q(\xi)=0, \lim _{\xi \rightarrow \infty} Z(\xi)=0 .
$$


More precisely, there exist constants $C_{1}, C_{2} \in \mathbb{C}$, such that for all $\varepsilon>0$ there exists $\xi_{0}$ large enough satisfying

$$
\left|W(\xi)-1-C_{1} e^{z_{0} \xi}-C_{2} e^{z_{0}^{*} \xi}\right|<\varepsilon \quad \text { for all } \xi>\xi_{0},
$$

and

$$
\lim _{\xi \rightarrow \infty} e^{\left|\lambda_{0}\right| \xi}\|(W-1, Q, Z)\|<\infty,
$$

where $\lambda_{0}:=\operatorname{Re}\left(z_{0}\right)$. Here, $z_{0}$ is the root of $\left(1-z^{2}\right)(2+z)+\frac{b}{a^{4}}$ with the largest negative real part and, for definiteness, with $\operatorname{Im}\left(z_{0}\right) \geq 0$. Moreover, $\left|\lambda_{0}\right|>1$ for all $a>0$.

Proof. (i): Lemma 3.9 implies that $\lim _{\xi \rightarrow \infty} \Phi(\xi)=0$ and $0 \leq W(\xi) \leq 2$ for all $\xi \in \mathbb{R}$. With the notation $Y:=(W-1, Q, Z)$, we write the last three equations of (19) as a linear system:

$$
\frac{d Y}{d \xi}=\left[\frac{A}{\Phi^{\frac{m}{3}}}+B(W)\right] Y
$$

where

$$
A=\left(\begin{array}{ccc}
0 & 1 & 0 \\
0 & 0 & 1 \\
\alpha & 0 & 0
\end{array}\right), B(W)=\left(\begin{array}{ccc}
-W & 0 & 0 \\
0 & \frac{2}{3}+\frac{m-3}{3} W & 0 \\
0 & 0 & \frac{1}{3}-\frac{m+3}{3} W
\end{array}\right) .
$$

We introduce $\tau(\xi) \in \mathbb{R}$ by $\tau(0)=0$ and $d \tau=\frac{d \xi}{\Phi^{\frac{m}{3}}}$. Then $\Phi^{\frac{m}{3}} \frac{d Y}{d \xi}=\frac{d Y}{d \tau}$ and $\lim _{\xi \rightarrow \infty} \tau(\xi)=\infty$, since $\lim _{\xi \rightarrow \infty} \Phi(\xi)=0$. In terms of $\tau$, (47) reads

$$
\frac{d Y}{d \tau}=\left[A+\Phi^{\frac{m}{3}} B\right] Y .
$$

For $\tau_{0}>0$ and $\tau \geq \tau_{0}$, we define the operator $U\left(\tau, \tau_{0}\right)$ by

$$
U\left(\tau_{0} ; \tau_{0}\right)=I \quad \text { and } \quad \frac{d}{d \tau} U\left(\tau ; \tau_{0}\right)=\left[A+\Phi^{\frac{m}{3}} B(\tau)\right] U\left(\tau ; \tau_{0}\right) .
$$

We note that the matrix $B$ is uniformly bounded in $\tau$, since $0 \leq W(\tau) \leq 2$. Here and in the following, by an abuse of notation, the functions $(\Phi, W, Q, Z)$ are written as functions both of $\xi$ and of $\tau$, with the obvious meaning. By classical ODE theory, $U\left(\tau ; \tau_{0}\right)$ is uniquely defined.

Since $\Phi(\tau) \rightarrow 0$ as $\tau \rightarrow \infty$, we also have $\left\|\Phi^{\frac{m}{3}}(\tau) B(\tau)\right\| \rightarrow 0$. By the continuous dependence of $U\left(\tau ; \tau_{0}\right)$ on the initial data at $\tau=\tau_{0}$, we get

$$
\lim _{\tau_{0} \rightarrow \infty} \sup _{\left\|\tau-\tau_{0}\right\| \leq M}\left\|U\left(\tau ; \tau_{0}\right)-e^{\left(\tau-\tau_{0}\right) A}\right\|=0 .
$$

The distinct eigenvalues $\lambda_{i} \in \mathbb{C}$ of $A$ are given by $\lambda_{i}^{3}=\alpha$, i.e.

$$
\lambda_{1}=\alpha^{\frac{1}{3}}, \quad \lambda_{2}=\left(-\frac{1}{2}+\frac{\sqrt{3}}{2} i\right) \alpha^{\frac{1}{3}}, \quad \lambda_{3}=\left(-\frac{1}{2}-\frac{\sqrt{3}}{2} i\right) \alpha^{\frac{1}{3}} .
$$


Then, by (50) and since $\Phi^{\frac{m}{3}} B$ is a uniformly bounded given matrix, any solution of (49) can be expressed as a linear combination of three solutions $Y^{k}(\tau)$, with

$$
c e^{\operatorname{Re} \lambda_{k} \tau-d \int_{\tau_{0}}^{\tau}\left\|\Phi(s)^{\frac{m}{3}} B(s)\right\| d s} \leq\left\|Y_{i}^{k}\left(\tau ; \tau_{0}\right)\right\| \leq C e^{\operatorname{Re} \lambda_{k} \tau+d \int_{\tau_{0}}^{\tau}\left\|\Phi(s)^{\frac{m}{3}} B(s)\right\| d s},
$$

here $k, i \in\{1,2,3\}$, for all $\tau \geq \tau_{0}$ for a sufficiently large $\tau_{0}$, and for positive constants $c, C, d>0$ (see e.g. [2]). Using that $\operatorname{Re}\left(\lambda_{1}\right)>0$, we conclude that $Y$ is a linear combination of only $Y^{2}$ and $Y^{3}$. In particular,

$$
c e^{-\frac{1}{2} \alpha^{1 / 3} \tau-d \int_{\tau_{0}}^{\tau} \Phi \frac{m}{3}(s) d s} \leq\left\|Y\left(\tau ; \tau_{0}\right)\right\| \leq C e^{-\frac{1}{2} \alpha^{1 / 3} \tau+d \int_{\tau_{0}}^{\tau} \Phi \frac{m}{3}(s) d s} .
$$

for positive constants $c, C, d>0$. Since $\lim _{\tau \rightarrow \infty} \Phi(\tau)=0$ for $0<m<4$, we obtain $\left\|Y\left(\tau ; \tau_{0}\right)\right\| \rightarrow 0$ as $\tau \rightarrow \infty$. In particular, assertion (42) holds.

We turn to the proof of (43). As before, the asymptotic behavior of $\Phi$ in the variable $\xi$ is easily obtained from the first equation (19), since we know that $W \rightarrow 1$ as $\xi \rightarrow \infty$. This implies that $\lim _{\xi \rightarrow \infty} e^{\frac{m-4}{m} \xi} \Phi(\xi)=a$, for some constant $a>0$ and obtain the first statement in (43).

To obtain the asymptotic behavior of $(W, Q, Z)$, we use the asymptotic behavior of $\Phi$ together with (51). For $\tau \geq 2 \tau_{0}$, the asymptotic behavior of $\Phi$ in $\xi$ and $\tau$ imply

$$
\frac{2 m}{(4-m) a^{\frac{m}{3}}} e^{\frac{1}{3 \alpha}\left(\xi-\xi_{0}\right)} \leq \tau-\tau_{0} \leq \frac{4 m}{(4-m) a^{\frac{m}{3}}} e^{\frac{1}{3 \alpha}\left(\xi-\xi_{0}\right)},
$$

for $\tau_{0}=\tau\left(\xi_{0}\right)$ sufficiently large. Moreover, we can estimate the integral in the exponents of (51) using (52) and the asymptotics of $\Phi$ to conclude that

$$
C_{2} \log \left(\tau-\tau_{0}\right) \leq \int_{\tau_{0}}^{\tau} \Phi^{\frac{m}{3}}(s) d s \leq C_{1} \log \left(\tau-\tau_{0}\right)
$$

for $C_{1}, C_{2}>0$. Using (52) and (53) in (51), we obtain that there exists $\xi_{0}$ large enough such that for all $\xi \geq \xi_{0}$ there exist positive constants with

$$
C_{2} e^{-2 \Lambda(\xi)} e^{d_{2}\left(\xi-\xi_{0}\right)} \leq\left\|Y\left(\xi ; \xi_{0}\right)\right\| \leq C_{1} e^{-\frac{1}{2} \Lambda(\xi)} e^{d_{1}\left(\xi-\xi_{0}\right)},
$$

where $\Lambda(\xi)=\frac{3}{2} \alpha^{\frac{4}{3}} a^{-\frac{m}{3}} e^{\frac{1}{3 \alpha}\left(\xi-\xi_{0}\right)}$. This implies the last statement in (43).

(ii): By Lemma 3.9, we have $\Phi(\xi) \rightarrow a \geq 0$ as $\xi \rightarrow \infty$ (we rename $K$ to $a$ for consistency with (12)). Let us show first that $a>0$. Arguing by contradiction, we assume that $a=0$, then we can apply the same argument as in the proof of (i) with $\alpha=b$, and in particular the estimate (51) also holds. This implies

$$
\int_{0}^{\infty}|1-W(\xi)| d \xi<\infty
$$

In view of the first equation in (19), this yields $W(\xi) \rightarrow 1$ as $\xi \rightarrow \infty$. On the other hand, integrating the first equation in (19), we get

$$
\Phi(\xi)=\Phi(0) \exp \left(\int_{0}^{\xi}(1-W(\eta)) d \eta\right)>C>0,
$$


uniformly for $\xi \in(0, \infty)$, but this means that $a>0$, which is a contradiction. Then $a>0$, and the first equation in (19) yields $W(\xi) \rightarrow 1$ as $\xi \rightarrow \infty$.

As in (47), $Y:=(W-1, Q, Z)$ satisfies

$$
\frac{d Y}{d \xi}=\frac{1}{\Phi^{\frac{4}{3}}} A Y+B(W) Y=: \frac{1}{a^{\frac{4}{3}}} A Y+B(1) Y+C(\xi) Y,
$$

where the matrices $A$ and $B$ are given by (48) (with $\alpha=b$ ). Since $\Phi^{\frac{4}{3}}(\xi) \rightarrow a^{\frac{4}{3}}$ and $W(\xi) \rightarrow 1$ as $\xi \rightarrow \infty$, the matrix $C(\xi)$ satisfies $\|C(\xi)\| \rightarrow 0$. For $\xi_{0} \in \mathbb{R}$ and $\xi \geq \xi_{0}$, we define the operator $U\left(\xi, \xi_{0}\right)$ by

$$
U\left(\xi_{0} ; \xi_{0}\right)=I \quad \text { and } \quad \frac{d}{d \xi} U\left(\xi ; \xi_{0}\right)=\left[\frac{1}{a^{\frac{4}{3}}} A+B(1)+C(\xi)\right] U\left(\xi ; \xi_{0}\right) \text {. }
$$

Since $\|C(\xi)\| \rightarrow 0$ as $\xi \rightarrow \infty$, by classical ODE theory we have

$$
\lim _{\xi_{0} \rightarrow \infty} \sup _{\left\|\xi-\xi_{0}\right\| \leq M}\left\|U\left(\xi ; \xi_{0}\right)-e^{\left(\xi-\xi_{0}\right)\left(A / a^{\frac{4}{3}}+B(1)\right)}\right\|=0
$$

for any $M>0$ fixed. In particular, $W(\xi) \rightarrow 1$ as $\xi \rightarrow \infty$ implies also that $Q(\xi), Z(\xi) \rightarrow 0$, since this is an isolated critical point of (57) with $C \equiv 0$. The exponential decay to this point is hence given by the roots with negative real part of the characteristic polynomial of $A / a^{\frac{4}{3}}+B(1)$, which is

$$
P_{a}(z)=P_{0}(z)+\frac{b}{a^{4}}, \quad \text { where } P_{0}(z):=\left(1-z^{2}\right)(2+z) .
$$

Clearly, $P_{a}$ always has a positive real root since $b / a^{4}>0$ and $z=1$ is the only positive root of $P_{0}$. Furthermore, since $P_{0}$ has two real negative roots and $P_{a}$ cannot have purely imaginary roots, it follows that $P_{a}$ has two roots with negative imaginary part for all $a>0$ (by continuity in $a$ ). Now $\lambda_{0}=\operatorname{Re}\left(z_{0}\right)<0$, the real part of the roots $z_{0} \in \mathbb{C}$ with the largest negative real part, controls the exponential decay in the variable $\xi$. Finally, we note that $\left|\lambda_{0}\right|>1$. If the roots are real, this is easy to check. If two of the roots are complex conjugates, this follows from the fact that the sum of the three roots is equal to -2 and one of them is real and positive.

We can now prove the main theorem:

Proof of Theorem [2.1. (i): From Lemma 3.2 and Lemma 3.3 we have $I_{0} \subset$ $(0, \sqrt{12 \alpha})$ and $I_{0} \neq \emptyset$. Then Proposition 3.10 gives the behavior of solutions for $\kappa \in I_{0}$ in terms of the variables (17). Changing back to the original variables we conclude that (12) holds since $e^{\frac{m-4}{m} \xi} \Phi(\xi)=\frac{f(y)}{y}$ for $y=e^{\xi}$.

(ii): Let $z_{0}$ be defined as in Proposition $\left[3.10\right.$ (ii) and let $z_{0}=\lambda_{0}+i \omega_{0}$ for $\omega_{0} \geq 0$. In view of (45) we have

$$
\left|(\ln \Phi)_{\xi}-g(\xi) e^{-\left|\lambda_{0}\right| \xi}\right|=o\left(e^{-\left|\lambda_{0}\right| \xi}\right)
$$


for $g(\xi)=\operatorname{Re}\left(K e^{-\omega_{0} i \xi}\right)=A \cos \left(\omega_{0} \xi\right)+B \sin \left(\omega_{0} \xi\right)$ for some $A, B \in \mathbb{R}$. Integration yields

$$
\ln \Phi-\ln a+\left(A^{\prime} \cos \left(\omega_{0} \xi\right)+B^{\prime} \sin \left(\omega_{0} \xi\right)\right) e^{-\left|\lambda_{0}\right| \xi}=o\left(e^{-\left|\lambda_{0}\right| \xi}\right)
$$

for some $A^{\prime}, B^{\prime} \in \mathbb{R}$. Since $\Phi=\frac{f}{y}$, we get

$$
f(y)=a y e^{\tilde{g}(y) y^{-\left|\lambda_{0}\right|}}+o\left(y e^{C y^{-\left|\lambda_{0}\right|}}\right)=a y+a \tilde{g}(y) y^{-\left|\lambda_{0}\right|+1}+o\left(y^{-\left|\lambda_{0}\right|+1}\right)
$$

for $\tilde{g}(y)=\left(A^{\prime} \cos \left(\omega_{0} \ln y\right)+B^{\prime} \sin \left(\omega_{0} \ln y\right)\right)$.

As a consequence of the asymptotic behavior of the solutions encoded in Proposition 3.10 we obtain also that the energy of solutions of the form (7) decreases in the following sense:

Corollary 3.11. (i) Let $m \in(0,4)$ and $h(x, t)$ be a solution of (11) of the form (77), where $f$ solves (10)-(12). Then, there exist constants $d_{0}, D_{0}>0$ such that

$$
-t^{\alpha} d_{0}<\frac{1}{2} \int_{-\infty}^{\infty}\left(h_{x}^{2}-h_{\mathrm{in}, x}^{2}\right) d x \leq-t^{\alpha} D_{0}
$$

(ii) Let $m=4$ and let $h(x, t)$ be a solution of (11) of the form (8) where $f$ solves (10)-(12). Then, there exist constants $d_{0}, D_{0}>0$ such that

$$
-e^{b t} d_{0}<\frac{1}{2} \int_{-\infty}^{\infty}\left(h_{x}^{2}-h_{\mathrm{in}, x}^{2}\right) d x \leq-e^{b t} D_{0} .
$$

Proof. We only give the proof of (i) since the proof of (ii) proceeds analogously. We hence assume $m \in(0,4)$. The dissipation formula reads:

$$
\frac{1}{2} \int_{-\infty}^{\infty}\left(h_{x}^{2}-h_{\mathrm{in}, x}^{2}\right) d x+\int_{0}^{t} \int_{-\infty}^{\infty} h^{m} h_{x x x}^{2} d x d t=0 .
$$

Changing to the self-similar variables in (63) we obtain

$$
\frac{1}{2} \int_{-\infty}^{\infty}\left(h_{\mathrm{in}, x}^{2}-h_{x}^{2}\right) d x=\frac{2 t^{\alpha}}{\alpha} \int_{0}^{\infty} f^{m} f_{y y y}^{2} d y .
$$

We now observe that there exist $d_{0}>0$ and $D_{0}>0$ such that

$$
d_{0}<\int_{0}^{\infty} f^{m} f_{y y y}^{2} d y \leq D_{0} .
$$

In view of the definitions of $\Phi, Z$ in (17) and by Proposition 3.10(i), we have $\lim _{y \rightarrow \infty} \frac{f}{y}=\lim _{\xi \rightarrow \infty} e^{\frac{4-m}{m} \xi} \Phi(\xi)=a$ and thus

$$
\lim _{y \rightarrow \infty} e^{K_{0} y^{\frac{1}{3 \alpha}}} y^{\frac{2(m-1)}{3}}\left|f_{y y y}\right|=\lim _{y \rightarrow \infty}\left(e^{K_{0} y^{\frac{1}{3 \alpha}}} y^{\frac{1}{3}} f^{\frac{2 m-3}{3}}\left|f_{y y y}\right|\right)\left(y^{\frac{2 m-3}{3}} f^{-\frac{2 m-3}{3}}\right)<\infty .
$$

Hence, there are $C, c, c^{\prime}>0$ and $y_{0}$ large enough with

$$
c \int_{y_{0}}^{\infty} e^{-2 K_{0} y^{\frac{1}{3 \alpha}}} y^{-\frac{1}{3 \alpha}} d y \leq \int_{0}^{\infty} f^{m}\left(f_{y y y}\right)^{2} d y \leq c^{\prime}+C \int_{y_{0}}^{\infty} e^{-2 K_{0} y^{\frac{1}{3 \alpha}}} y^{-\frac{1}{3 \alpha}} d y .
$$

This implies the existence of $d_{0}>0$ and $D_{0}>0$ such that (61) holds. 
Acknowledgments: The authors acknowledge the financial support of the German Science Foundation (DFG) through the project CRC 1060 The mathematics of emergent effects, of the Spanish Government through the MINECO project MTM2014-53145-P, and of the Basque Government through the Research Group grant IT641-13.

\section{A Merging of two droplets}

In this section we briefly outline the formal asymptotics that corresponds to the merging of two droplets in the case $0<m<4$. Namely, we consider (1) with initial data

$$
h_{\text {in }}(x)=\left(a|x|-b x^{2}\right)_{+}
$$

for $a, b>0$. Notice that $h_{\mathrm{in}}$ is a stationary solution of (11) representing two identical droplets with a contact touch-down point at $x=0$. Also the cone conditions (5) - (6) are clearly satisfied at the contact point $x=0$. We assume that lifting takes place in the self-similar way (7) at $x=0$. To leading order as $t \rightarrow 0^{+}$, the solution is then given by the self-similar solution of (10)-(12). The behavior (12), with exponential corrections, gives the matching into $h_{\text {in }}(x)$ for small times. In order to derive the next order correction, we write

$$
h(x, t)=t^{\alpha} f\left(\frac{x}{t^{\alpha}}, \log t\right) .
$$

Inserting this ansatz into (11) and with the notation $y=\frac{|x|}{t^{\alpha}}, \tau=\log t$, we get

$$
f_{\tau}+\alpha\left(f-y f_{y}\right)+\left(f^{m} f_{y y y}\right)_{y}=0 .
$$

To be consistent with (64), we impose the matching condition

$$
t^{\alpha} f(y, \tau) \sim\left(a|x|-b x^{2}\right)_{+}=t^{\alpha}\left(a|y|-b e^{\alpha \tau} y^{2}\right)_{+}
$$

for $|y| \gg 1, \tau \rightarrow-\infty, e^{\tau \alpha} y=|x| \ll 1$. Let us denote by $f_{0}$ the solution to (10)-(12). In order to determine the next order correction $f_{1}$, we make the ansatz

$$
f(y, \tau)=f_{0}(y)+f_{1}(y, \tau) .
$$

In view of (65) and since $\frac{f_{0}(y)}{y} \rightarrow a$ for $|y| \rightarrow \infty$, this yields the following matching condition for $f_{1}$ :

$$
f_{1}(y, \tau) \sim-b e^{\alpha \tau} y^{2} \quad \text { as }|y| \gg 1, \quad \tau \rightarrow-\infty .
$$

This suggests that we look for functions $f_{1}$ of the form

$$
f_{1}(y, \tau)=e^{\alpha \tau} P(y),
$$


where $P$ has the asymptotic behavior

$$
P(y) \sim-b y^{2} \quad \text { as } \quad|y| \gg 1
$$

and where $P$ solves the leading order balance equation

$$
2 \alpha P-y P_{y}+\left(f_{0}^{m} P_{y y y}\right)_{y}+m\left(f_{0}^{m-1} f_{0, y y y} P\right)_{y}=0 .
$$

Assuming that there is a solution of (67)- 68 for a given $b$, we obtain the next order correction by solving this problem and (66).

\section{References}

[1] J.W. Barrett, J.F. Blowey, and H. Garcke. Finite element approximation of a fourth order nonlinear degenerate parabolic equation. Numer. Math., 80(4):525-556, 1998.

[2] R. Bellman. Stability theory in differential equations. Dover Publications, Inc., New York, 1969.

[3] E. Beretta, M. Bertsch, and R. Dal Passo. Nonnegative solutions of a fourth-order nonlinear degenerate parabolic equation. Arch. Rational Mech. Anal., 129:175-200, 1995.

[4] F. Bernis and A. Friedman. Higher order nonlinear degenerate parabolic equations. J. Differential Equations, 83:179-206, 1990.

[5] F. Bernis, J. Hulshof, and J. R. King. Dipoles and similarity solutions of the thin film equation in the half-line. Nonlinearity, 13:413-439, 2000.

[6] F. Bernis, L. A. Peletier, and S. M. Williams. Source type solutions of a fourth order nonlinear degenerate parabolic equation. Nonlinear Anal., 18:217-234, 1992 .

[7] A. L. Bertozzi and M. Pugh. The lubrication approximation for thin viscous films: regularity and long-time behavior of weak solutions. Comm. Pure Appl. Math., 49:85-123, 1996.

[8] A.L. Bertozzi. The mathematics of moving contact lines in thin liquid films. Notices Amer. Math. Soc., 45(6):689-697, 1998.

[9] M. Bertsch, R. Dal Passo, H. Garcke, and G. Grün. The thin viscous flow equation in higher space dimensions. Adv. Differential Equations, 3:417$440,1998$.

[10] M. Bertsch, L. Giacomelli, and G. Karali. Thin-film equations with "partial wetting" energy: existence of weak solutions. Phys. D, 209:17-27, 2005.

[11] P. Constantin, T. F. Dupont, R. E. Goldstein, L. P. Kadanoff, M. J. Shelley, and S. Zhou. Droplet breakup in a model of the Hele-Shaw cell. Phys. Rev. E, 47:4169-4181, Jun 1993. 
[12] R. Dal Passo, H. Garcke, and G. Grün. On a fourth-order degenerate parabolic equation: global entropy estimates, existence, and qualitative behavior of solutions. SIAM J. Math. Anal., 29:321-342 (electronic), 1998.

[13] J. J. Duistermaat and J. A. C. Kolk. Distributions. Cornerstones. Birkhäuser Boston, Inc., Boston, MA, 2010. Theory and applications, Translated from the Dutch by J. P. van Braam Houckgeest.

[14] E. B. Dussan and S. H. Davis. On the motion of a fluid-fluid interface along a solid surface. J. Fluid Mech., 65:71-95, 1974.

[15] R. Ferreira and F. Bernis. Source-type solutions to thin-film equations in higher dimensions. European J. Appl. Math., 8:507-524, 1997.

[16] L. Giacomelli, M. V. Gnann, H. Knüpfer, and F. Otto. Well-posedness for the Navier-slip thin-film equation in the case of complete wetting. $J$. Differential Equations, 257:15-81, 2014.

[17] L. Giacomelli, M. V. Gnann, and F. Otto. Regularity of source-type solutions to the thin-film equation with zero contact angle and mobility exponent between 3/2 and 3. European J. Appl. Math., 24:735-760, 2013.

[18] L. Giacomelli and H. Knüpfer. A free boundary problem of fourth order: classical solutions in weighted Hölder spaces. Comm. Partial Differential Equations, 35:2059-2091, 2010.

[19] L. Giacomelli, H. Knüpfer, and F. Otto. Smooth zero-contact-angle solutions to a thin-film equation around the steady state. J. Differential Equations, 245:1454-1506, 2008.

[20] L. Giacomelli and A. Shishkov. Propagation of support in one-dimensional convected thin-film flow. Indiana Univ. Math. J., 54(4):1181-1215, 2005.

[21] M. V. Gnann. On the regularity for the Navier-slip thin-film equation in the perfect wetting regime. Arch. Ration. Mech. Anal., 222(3):1285-1337, 2016.

[22] H. P. Greenspan. Motion of a small viscous droplet that wets a surface. J. Fluid Mech., 84:125-143, 1978.

[23] G. Grün. Droplet spreading under weak slippage - existence for the Cauchy problem. Comm. Partial Diff. Eq., 29(11-12):1697-1744, 2004.

[24] C. Huh and L.E. Scriven. Hydrodynamic model of steady movement of a solid/liquid/fluid contact line. J. Coll. Int. Sc., 35:85-101, 1971.

[25] D. John. On uniqueness of weak solutions for the thin-film equation. $J$. Differential Equations, 259(8):4122-4171, 2015.

[26] H. Knüpfer. Navier slip thin-film equation for partial wetting. Comm. Pure Appl. Math., 64:1263-1296, 2011. 
[27] H. Knüpfer and N. Masmoudi. Well-posedness and uniform bounds for a nonlocal third order evolution operator on an infinite wedge. Comm. Math. Phys., 320(2):395-424, 2013.

[28] H. Knüpfer and N. Masmoudi. Darcy's flow with prescribed contact angle: well-posedness and lubrication approximation. Arch. Ration. Mech. Anal., 218(2):589-646, 2015.

[29] A. Oron, S. Davis, and S. Bankoff. Long-scale evolution of thin liquid films. Rev. Mod. Phys., 69(3):931-980, 1997.

[30] F. Otto. Lubrication approximation with prescribed nonzero contact angle. Comm. Partial Differential Equations, 23:2077-2164, 1998.

[31] O. Reynolds. On the theory of lubrication and its application to Mr. Beauchamp Tower's experiments, including an experimental determination of the viscosity of olive oil. Proc. R. Soc. London, 40:191-203, 1886. 
This figure "evolution3.png" is available in "png" format from: http://arxiv.org/ps/1708.00243v1 